\title{
ESTUDO DA FREQUÊNCIA DE ALTERAÇÕES COMPORTAMENTAIS EM FELINOS DOMÉSTICOS
}

Study of the frequency of behavioral changes in domestic cats

Estudio de la frecuencia de los cambios de comportamiento en gatos domésticos

\section{Camila Moura de Lima* ${ }^{*}$, Caroline Xavier Grala², Gustavo Antônio Boff ${ }^{1}$, Mariana Cristina Hoeppner Rondelli ${ }^{3}$, Márcia de Oliveira Nobre ${ }^{3}$}

${ }^{1}$ Faculdade de Veterinária, Discente do curso de mestrado acadêmico em veterinária, Universidade Federal de Pelotas, Capão do Leão, Brasil.

${ }^{2}$ Faculdade de Veterinária, Discente do curso de Graduação em medicina veterinária, Universidade Federal de Pelotas, Capão do Leão, Brasil.

${ }^{3}$ Departamento de Clínicas Veterinária, Professor adjunto de clínica médica de pequenos animais, Universidade Federal de Pelotas, Capão do Leão, Brasil.

*Correspondência: Faculdade de Veterinária, Universidade Federal de Pelotas, campus universitário s/n, Capão do Leão, Rio Grande do Sul, Brasil. CEP:96160-000.e-mail camila.moura.lima@hotmail.com

Artigo recebido em 20/12/2019 aprovado em 06/11/2020 publicado em 04/03/2021.

\section{RESUMO}

A interação homem-animal ocorre há muitos anos e devido a isso existe o aumento contínuo na população de animais. Atualmente, há pouco espaço e tempo disponibilizado devido à rotina dos tutores, logo a espécie felina tende a se adaptar mais facilmente a esse ambiente. Entretanto, se não houver recursos ambientais onde o animal possa expressar seu comportamento natural haverá o surgimento de alterações comportamentais. O objetivo deste estudo foi relatar as alterações comportamentais nos felinos domésticos domiciliados. Foi elaborado um questionário online contendo 31 questões objetivas. As repostas foram anônimas e consideravam os dados gerais dos tutores e felinos, a forma de distribuição do ambiente e questões relacionadas a alterações comportamentais. Foi demonstrado, que os fatores relacionados à falta de manejo como, o baixo período disponibilizado para a interação tutor-animal, baixa frequência de troca de brinquedos e o elevado número de animais por residência podem estar relacionados ao aparecimento de alterações consideradas indesejáveis pelos tutores. Assim é de grande importância orientar os tutores sobre as características etológicas da espécie, com o intuito de proporcionar qualidade de vida e bem-estar aos felinos e aos tutores. Dessa forma, conclui-se que na população estudada houve uma maior frequência de destruição de objetos com arranhadura e eliminação inapropriada, sendo essas as principais alterações comportamentais encontradas nos felinos estudados.

Palavras-chave: Gato, comportamento natural, estresse, alteração comportamental, bem-estar.

\section{ABSTRACT}

Human-animal interaction has been going on for many years and because of this there is a continuous increase in the population of animals. Currently, there is little space and time available due to the tutors' routine, so the feline species tends to adapt more easily to this environment. However, if there are no environmental resources where the animal can express its natural behavior, there will be behavioral changes. The aim of this study was to report behavioral changes in domesticated domestic cats. An online questionnaire was prepared containing 31 objective questions. It has been shown that factors related to lack of management, such as the low time available for the tutoranimal interaction, low frequency of changing toys and the high number of animals per residence can be related to the appearance of changes considered undesirable by tutors. Thus, it is of great importance to guide tutors on the ethological characteristics of the species, in order to provide quality of life and well-being to felines and tutors. Thus, it is concluded that in the studied population there was a greater frequency of destruction of objects with scratching and inappropriate elimination, these being the main behavioral changes found in the studied cats.

Keywords: Cat, natural behavior, stress, behavioral change, well-being. 


\section{RESUMEN}

La interacción entre humanos y animales ha estado ocurriendo durante muchos años y, debido a esto, hay un aumento continuo en la población animal. Actualmente, hay poco espacio y tiempo disponible debido a la rutina de los tutores, por lo que la especie felina tiende a adaptarse más fácilmente a este entorno. Sin embargo, si no existen recursos ambientales donde el animal pueda expresar su comportamiento natural, habrá cambios de comportamiento. El objetivo de este estudio fue informar cambios de comportamiento en gatos domésticos domesticados. Se preparó un cuestionario en línea con 31 preguntas objetivas. Las respuestas fueron anónimas y consideraron los datos generales de los guardianes y felinos, la forma de distribución del entorno y cuestiones relacionadas con cambios de comportamiento. Se ha demostrado que factores relacionados con la falta de manejo, como el escaso tiempo disponible para la interacción tutor-animal, la baja frecuencia de cambio de juguetes y el elevado número de animales por residencia pueden estar relacionados con la aparición de cambios considerados indeseables por los tutores. Por ello, es de gran importancia orientar a los tutores sobre las características etológicas de la especie, con el fin de brindar calidad de vida y bienestar a los felinos y tutores. Así, se concluye que en la población estudiada hubo una mayor frecuencia de destrucción de objetos con rascado y eliminación inadecuada, siendo estos los principales cambios de comportamiento encontrados en los gatos estudiados.

Descriptores: Gato, comportamiento natural, estrés, cambio de comportamiento, bienestar.

\section{INTRODUÇÃO}

A interação homem-animal ocorre há muitos anos. Esse vínculo cada vez mais é fortalecido devido aos benefícios, que os animais podem proporcionar as pessoas a nível fisiológico, psicológico e social (FARACO e SOARES, 2013). Devido a isso há o aumento contínuo na população de animais de estimação, sendo o Brasil o segundo maior do mundo em população de cães, gatos e aves canoras e ornamentais (IBGE, 2013). Atualmente, há pouco espaço e tempo disponibilizado devido à rotina dos tutores, $\log$ a espécie felina tende a se adaptar mais facilmente quando comparada aos cães (GENARO, 2013).

Entretanto, se não houver recursos ambientais onde o animal possa expressar seu comportamento natural haverá o surgimento de situações que desencadeie o estresse e a ansiedade. Logo contribuirá para o aparecimento de alterações comportamentais e até mesmo o aparecimento de doenças em vários sistemas, dentre eles, cardiovascular, locomotor, urinário e digestivo (AAFP/IFSM, 2013; FARACO e SOARES, 2013).

Nesse contexto, a medicina comportamental possui grande relevância na medicina veterinária, pois as alterações consideradas indesejáveis por parte dos tutores podem contribuir para situações estressantes nas famílias gerando a destruição do vínculo, punição inadequada, abandono e até mesmo a eutanásia (AAFP/IFSM, 2013; SCHOT et al., 2016).

Em vista disso, o objetivo deste trabalho foi relatar as alterações comportamentais nos felinos domésticos domiciliados.

\section{MATERIAIS E MÉTODOS}

Para a realização deste estudo foi elaborado um questionário contendo 31 questões objetivas por colaboradores de um projeto de pesquisa vinculado ao grupo de pesquisa, ensino e extensão em clínica médica de pequenos animais da Universidade Federal de Pelotas. O questionário foi disponibilizado na internet, sendo direcionado especialmente para tutores de felinos domésticos domiciliados, independente do local de domicílio (SOUZA \& MEDEIROS, 2016; KRUG et al., 2019). As perguntas foram disponibilizadas em um período de cinco dias em duas plataformas digitais (Facebook e instagram) no seguinte link < https://forms.gle/hwb83xRk5ouyHkK4>.

O questionário foi dividido em três secções, sendo a primeira uma área destinada a uma breve 
apresentação do objetivo do estudo e a solicitação para a participação da pesquisa, após a ciência por parte do tutor havia o direcionamento para as questões. A segunda secção compreendia os dados do tutor, apesar das respostas serem anônimas, consideravam a idade dos tutores, sexo, tipo de moradia e quantas pessoas residiam no local.

A última secção correspondia aos dados dos felinos onde foi considerada a raça, sexo, idade, peso, estado reprodutivo, tipo de alimentação e quantas vezes era fornecido ao dia, nível de atividade física e a forma de distribuição do ambiente como, separação do local de alimentação, vasilha sanitária e descanso. E caso possuísse outros animais se havia mais de uma vasilha para comer e quantas caixas de areia eram disponibilizadas por animal. Bem como, se tinha disponível arranhador vertical, local alto para ficar, brinquedos e a frequência de troca e ainda se conseguia locomover-se livremente pela casa.

Também havia perguntas direcionadas ao tempo e forma de interação tutor-animal e uma área direcionada a alterações comportamentais como, demonstração de agressividade na hora das brincadeiras, com outros animais e pessoas. Bem como, se já houve episódios de eliminação inapropriada, destruição de móveis/objetos, vocalização excessiva e qual a reação do felino frente à chegada de pessoas diferentes. Após a disponibilização do questionário online, os dados foram analisados e avaliados com a estatística descritiva por meio da frequência de cada resposta pelo programa GraphPad prism 7.0.

\section{RESULTADOS E DISCUSSÃO}

Com base na divulgação do questionário online, em plataformas digitais, foram obtidas 264 respostas. Esse instrumento foi útil, pois foi possível abranger uma população de pessoas e felinos de diferentes regiões. Em relação aos dados dos tutores foi possível verificar, que houve um maior predomínio na faixa etária de 21 e 30 anos com 48,1\% ( $n=127$ ), gênero feminino $89,4 \% \quad(n=236)$, morando em casa $67 \%$ $(\mathrm{n}=177)$, residindo com até duas pessoas $44,7 \%$ $(\mathrm{n}=118)$ e $84,1 \%(\mathrm{n}=222)$ possuíam mais de um animal, (Tab.1). Os resultados obtidos estão de acordo com a literatura, que relata um maior predomínio de tutores jovens, do sexo feminino com duas ou mais pessoas. Embora os resultados tenham indicado que a maior taxa de moradia era em casa a literatura relata como principal residência o apartamento. Porém o tipo de moradia pode variar de acordo com a região, pois em regiões com maior urbanização o felino tem sido o animal de companhia preferido, justamente por possuir a tendência de se adaptar mais facilmente a espaços menores (GENARO; ALVES, 2017).

Tabela 1. Dados gerais referentes, aos tutores de felinos, considerando à faixa etária, gênero, tipo de moradia, número de pessoas e animais por residência.

\begin{tabular}{llll}
\hline Variável & Grupos & n & \% \\
\hline Faixa etária & $\geq 20$ anos & 14 & 5,3 \\
& 21 a 30 anos & 127 & 48,1 \\
& 30 a 40 anos & 56 & 21,2 \\
& 41 a 50 anos & 43 & 16,3 \\
& Acima de 50 anos & 24 & 9,1 \\
\hline Gênero & Feminino & 236 & 89,4 \\
& Masculino & 28 & 10,6 \\
\hline Tipo de moradia & Casa & 177 & 67 \\
& Apartamento & 87 & 33 \\
\hline Número de & Mora sozinho & 33 & 12,5 \\
moradores & Até duas pessoas & 118 & 44,7 \\
& Mais de duas & 113 & 42,8 \\
& pessoas & & \\
\hline Número de animais & Apenas um & 42 & 15,9 \\
& Mais de um & 222 & 84,1 \\
\hline
\end{tabular}


Em relação aos dados dos felinos foi possível verificar uma maior ocorrência de animais jovens na faixa etária de 1 a 5 anos 72,3\% (n=191), fêmeas 64,7\% $(\mathrm{n}=171)$, sem raça definida $93,2 \%(\mathrm{n}=246)$, castrados $87,1 \%(\mathrm{n}=230)$, com peso médio de $4,3 \mathrm{~kg}$,

$67 \%(\mathrm{n}=177)$ que possuíam acesso à rua e $80,3 \%$ $(n=212)$ foram adotados com menos de 1 ano (Tab.2). Segundo Paz (2017) relata uma maior frequência em fêmeas, sem raça definida, castradas. Entretanto, esses resultados podem variar de acordo com a localização, condições socioeconômicas dos tutores e a preferência por uma determinada raça (QUESSADA et al., 2014).

Tabela 2. Dados gerais dos felinos referentes à faixa etária, sexo, raça, estado reprodutivo, acesso à rua e a idade que foi adotado.

\begin{tabular}{|c|c|c|c|}
\hline Variável & Grupos & $\mathbf{n}$ & $\%$ \\
\hline \multirow[t]{3}{*}{ Faixa etária } & 1 a 5 anos & 191 & 72,3 \\
\hline & 6 a 10 anos & 60 & 22,7 \\
\hline & Acima de 10 anos & 13 & 4,9 \\
\hline \multirow[t]{2}{*}{ Sexo } & Macho & 93 & 35,3 \\
\hline & Fêmea & 171 & 64,7 \\
\hline \multirow[t]{5}{*}{ Raça } & Sem raça definida & 246 & 93,2 \\
\hline & Siamês & 9 & 3,4 \\
\hline & Persa & 5 & 1,9 \\
\hline & Angorá & 2 & 0,7 \\
\hline & $\begin{array}{l}\text { Pêlo curto } \\
\text { americano }\end{array}$ & 2 & 0,7 \\
\hline \multirow[t]{2}{*}{$\begin{array}{l}\text { Estado } \\
\text { reprodutivo }\end{array}$} & Castrado (a) & 230 & 87,1 \\
\hline & Não castrado (a) & 34 & 12,9 \\
\hline \multirow[t]{2}{*}{ Acesso à rua } & Sim & 177 & 67 \\
\hline & Não & 87 & 33 \\
\hline \multirow[t]{5}{*}{$\begin{array}{l}\text { Idade que foi } \\
\text { adotado }\end{array}$} & $>1$ ano & 212 & 80,3 \\
\hline & 1 a 5 anos & 33 & 12,5 \\
\hline & 5 a 10 anos & 3 & 1,1 \\
\hline & Acima de 10 anos & 10 & 3,8 \\
\hline & Não soube opinar & 6 & 2,3 \\
\hline
\end{tabular}

Em relação à forma de distribuição do ambiente e os hábitos dos felinos foi possível verificar, que a maioria dos tutores fornecia ração seca e petiscos $50 \%(\mathrm{n}=132)$, alimentação à vontade 60,6\% $(\mathrm{n}=160)$, os animais possuíam áreas distintas para a alimentação, vasilha sanitária e local de descanso 81,8\% ( $\mathrm{n}=216)$. Bem como, tutores com mais de um gato revelaram, que não disponibilizavam mais de uma vasilha sanitária por animal 45,1\% ( $\mathrm{n}=119)$. Já de acordo com a presença de brinquedos $67 \%(\mathrm{n}=177)$ e $55,3 \%(\mathrm{n}=146)$ não trocavam com frequência os brinquedos, $56,1 \%$ $(\mathrm{n}=148)$ possuíam arranhador vertical e $42,5 \%(\mathrm{n}=112)$ não, $80,3 \%(n=212)$ felinos possuíam local alto para ficar. Ademais, a maior parte dos tutores revelaram disponibilizar até uma hora para a interação com o animal 53,4\% (n=141) e o período de maior interação foi o da noite com 81,8\% ( $\mathrm{n}=216)$. Além disso, foi possível verificar, que $48,9 \%$ (n=129) consideravam os animais ativos em relação ao nível de atividade física e 99,2\% ( $\mathrm{n}=262)$ dos felinos conseguiam se locomover livremente pela residência, (Tab.3).

As necessidades ambientais dos felinos não compreendem somente o local onde ele está inserido, mas também vale ressaltar a importância da interação social desses animais (WESTROPP, 2006). Dessa forma, salienta-se a importância do conhecimento do comportamento natural dos felinos, a fim de buscar recursos que atendam as necessidades etológicas e ambientais como, um local seguro, ambientes distintos para lazer, descansar, alimentar, afiar as garras, número correto de vasilha sanitária por animal, estimular o instinto predatório através de brincadeiras e proporcionar um momento de interação homem-animal (AAFP/IFSM, 2013). 
Tabela 3. Forma de distribuição do ambiente, frequência e tipo de alimento, presença e frequência de troca de brinquedos, período de interação tutor-animal e nível de atividade física do animal.

\begin{tabular}{|c|c|c|c|}
\hline Variável & Grupos & $\mathbf{n}$ & $\%$ \\
\hline \multirow[t]{3}{*}{ Tipo de alimento } & Somente ração & 120 & 45,4 \\
\hline & Ração e petisco & 132 & 50 \\
\hline & Ração e comida caseira & 12 & 4,6 \\
\hline \multirow[t]{4}{*}{ Frequência de fornecimento do alimento } & $\grave{A}$ vontade & 160 & 60,6 \\
\hline & $1 \mathrm{vez}$ ao dia & 1 & 0,4 \\
\hline & 2 vezes ao dia & 47 & 17,8 \\
\hline & Mais de duas vezes ao dia & 56 & 21,2 \\
\hline \multirow[t]{5}{*}{ Distribuição do ambiente } & Áreas distintas & 216 & 81,8 \\
\hline & Não separa alimentação da vasilha sanitária & 24 & 9,1 \\
\hline & Não separa alimentação do local de descanso & 13 & 4,9 \\
\hline & Não separa local de descanso da vasilha sanitária & 3 & 1,1 \\
\hline & Não opinaram & 8 & 3 \\
\hline \multirow[t]{3}{*}{ Mais de uma vasilha sanitária por animal } & Sim & 79 & 29,9 \\
\hline & Não & 119 & 45,1 \\
\hline & Não opinaram & 66 & 25 \\
\hline \multirow[t]{2}{*}{ Presença de brinquedo } & Sim & 177 & 67 \\
\hline & Não & 87 & 33 \\
\hline \multirow[t]{3}{*}{ Frequência de troca de brinquedo } & Sim & 80 & 30,3 \\
\hline & Não & 146 & 55,3 \\
\hline & Não opinaram & 38 & 14,4 \\
\hline \multirow[t]{3}{*}{ Possui arranhador } & Sim & 148 & 56,1 \\
\hline & Não & 112 & 42,5 \\
\hline & Não opinaram & 4 & 1,5 \\
\hline \multirow[t]{2}{*}{ Possui local alto pra ficar } & Sim & 212 & 80,3 \\
\hline & Não & 52 & 19,7 \\
\hline \multirow[t]{5}{*}{ Tempo disponibilizado para a interação } & Até 1 hora & 141 & 53,4 \\
\hline & Acima de 1 hora & 103 & 39 \\
\hline & Não soube opinar & 5 & 1,9 \\
\hline & Sempre quando ele quer & 10 & 3,8 \\
\hline & Nenhum tempo disponível & 5 & 2,3 \\
\hline \multirow[t]{4}{*}{ Período de maior interação } & Manhã & 6 & 2,3 \\
\hline & Tarde & 16 & 6,1 \\
\hline & Noite & 216 & 81,8 \\
\hline & Não opinaram & 26 & 9,8 \\
\hline \multirow[t]{3}{*}{ Nível de atividade física } & Pouco ativo & 86 & 32,6 \\
\hline & Ativo & 129 & 48,9 \\
\hline & Muito ativo & 49 & 18,5 \\
\hline \multirow[t]{2}{*}{ O felino locomove-se livremente pela casa } & Sim & 262 & 99,2 \\
\hline & Não & 2 & 0,08 \\
\hline
\end{tabular}

Em relação ao comportamento dos felinos e as alterações comportamentais verificou-se que a grande parte dos felinos $49,2 \%(\mathrm{n}=130)$ sente-se a vontade quando chegam pessoas diferentes na residência,
82,9\% ( $\mathrm{n}=219)$ não possuíam o hábito de vocalizar, $70,5 \%$ ( $\mathrm{n}=186$ ) ficavam próximo do tutor no período de interação. Bem como, 69,7\% ( $n=184), 77,7 \%$ $(n=205)$ e $51,5 \%(n=136)$ não eram agressivos durante 
as brincadeiras, com outras pessoas e animais, respectivamente. Já $77,3 \%(n=204)$ revelaram que o animal já destruiu algum móvel como, cadeira e sofá e $50,7 \% \quad(n=134)$ já tiveram algum episódio de eliminação inapropriada (Tab.4).

Contudo, se as necessidades etológicas e ambientais não forem cumpridas pode-se promover o aparecimento de ansiedade e estresse. Logo, em situações estressantes prolongadas o aumento do cortisol pode desencadear efeitos negativos a saúde do animal como, alterações metabólicas e comportamentais (MAJZOUB, 2006; FARACO e SOARES, 2013). Porém, deve-se possuir uma visão ampla da trajetória do animal, levando em consideração também o temperamento do felino, que pode estar correlacionado a genética ou a fase inicial de socialização, que ocorre entre 3 e 9 semanas de vida (BUFFINGTON, 2012; AMAT, 2016).

De acordo com os resultados obtidos neste estudo pode-se verificar que houve uma maior frequência 77,3\% (n=204) na destruição de objetos com arranhadura e 50,7\% $(n=134)$ eliminação inapropriada. A arranhadura é um comportamento natural e pode ser considerado indesejável pela percepção do tutor, como foi visto neste estudo. Já a eliminação inapropriada pode ser explicada, pela não disponibilidade do correto número de vasilhas sanitárias por animal, pelo elevado número de animais e também pela interação social, onde os animais podem demonstrar esse comportamento devido à marcação territorial (NEILSON, 2004).

Os resultados obtidos neste estudo podem ser explicados devido ao baixo período disponibilizado para a interação tutor-animal, baixa frequência de troca de brinquedos e o elevado número de animais por residência podem ser desencadeantes para o aparecimento de alterações consideradas indesejáveis pelos tutores. Devido a isso sugere-se a realização estudos futuros, a fim de identificar as possíveis causas e correlacionar com as alterações comportamentais dos felinos domésticos. Dessa maneira, é de grande importância informar os tutores sobre as características e necessidades da espécie e também informar os locais adequados para a realização desses comportamentos, a fim de promover e atender as necessidades etológicas e ambientais.

Tabela 4. Comportamento dos felinos referente a chega de pessoas estranhas, frequência de vocalização excessiva, interação humano-animal, agressividade nas brincadeiras, com outras pessoas e animais, destruição de moveis e eliminação inapropriada.

\begin{tabular}{|c|c|c|c|}
\hline Variável & Grupos & $\mathbf{n}$ & $\%$ \\
\hline \multirow[t]{3}{*}{$\begin{array}{l}\text { Comportamento } \\
\text { frente às visitas }\end{array}$} & $\begin{array}{l}\text { Sente-se à } \\
\text { vontade }\end{array}$ & 130 & 49,2 \\
\hline & Esconde-se & 93 & 35,2 \\
\hline & Não interage & 41 & 15,3 \\
\hline \multirow[t]{2}{*}{$\begin{array}{l}\text { Frequência de } \\
\text { vocalização }\end{array}$} & Sim & 45 & 17,1 \\
\hline & Não & 219 & 82,9 \\
\hline \multirow[t]{4}{*}{$\begin{array}{l}\text { Quanto está em casa o } \\
\text { felino fica onde }\end{array}$} & Próximo & 186 & 70,5 \\
\hline & No colo & 55 & 20,8 \\
\hline & Dormindo & 18 & 6,8 \\
\hline & Não opinaram & 5 & 1,9 \\
\hline \multirow[t]{2}{*}{$\begin{array}{l}\text { Agressividade nas } \\
\text { brincadeiras }\end{array}$} & Sim & 80 & 30,3 \\
\hline & Não & 184 & 69,7 \\
\hline \multirow[t]{4}{*}{$\begin{array}{l}\text { Agressividade com } \\
\text { outras pessoas }\end{array}$} & Sim & 10 & 3,8 \\
\hline & Não & 205 & 77,7 \\
\hline & Às vezes & 45 & 17 \\
\hline & Não opinaram & 4 & 1,5 \\
\hline \multirow[t]{3}{*}{$\begin{array}{l}\text { Agressividade com } \\
\text { outros animais }\end{array}$} & Sim & 48 & 18,2 \\
\hline & Não & 136 & 51,5 \\
\hline & Às vezes & 80 & 3 \\
\hline \multirow[t]{2}{*}{$\begin{array}{l}\text { Destruição de móveis } \\
\text { com arranhadura }\end{array}$} & Sim & 204 & 77,3 \\
\hline & Não & 60 & 22,7 \\
\hline \multirow[t]{2}{*}{$\begin{array}{l}\text { Eliminação } \\
\text { inapropriada }\end{array}$} & Sim & 134 & 50,7 \\
\hline & Não & 130 & 49,3 \\
\hline
\end{tabular}




\section{CONCLUSÃO}

Portanto, conclui-se que houve uma maior frequência de destruição de objetos com arranhadura e eliminação inapropriada, sendo essas as principais alterações comportamentais encontradas nos felinos estudados.

\section{AGRADECIMENTO}

Agradecimento ao capes pela concessão da bolsa contribuindo, dessa forma, para o desenvolvimento da pesquisa.

Todos os autores declararam não haver qualquer potencial conflito de interesses referente a este artigo.

\section{REFERÊNCIAS}

AAFP.; ISFM. Feline Environmental Needs Guidelines. Jour. of Fel. Med. and Surg. v.15,p.219230,2013.

ALVES, R.S.; BARBOSA, R.C.C.; GHEREN, M.W.; SILVA, L.E.; SOUZA, H.JM. Frequência e fatores de risco da obesidade em uma população de gatos domésticos do Rio de Janeiro. Braz. Jou. of Vet. Med., v.39, n.1, p.33-45, 2017.

AMAT, M.; CAMPS, T.; MANTECA, X. Stress in owned cats: behavioural changes and welfare implications. Jou. of Fel. Med. and Sug., v. 18, n.8, p.577-586,2016.

BUFFINGTON, C.A. Conhece as recomendações da AAFP-AAHA para as diversas fases da vida dos felinos?. Vet. Med. 2012 Disponível em: https://www.hospitaldosanimais.com/Portals/4/new sletter/HDA_AAFP_02.pdf. Acesso: 28 out 2019.

FARACO, C.B.; SOARES, G.M. Fundamentos do comportamento canino e felino. $1^{\text {a }}$ ed., MedVet, 242 p., 2013.
GENARO, G. Aplicação de conceitos básicos em etologia na clínica médica veterinária felina. Rev. de educ. cont. em med. Vet. e zoot. v.11, n.1,p.32-37, 2013.

IBGE. População de animais de estimação no Brasil.p.1-7,2013.

KRUG, F.D.M.; TILMANN, M.T.; PIÑEIRO, M.B.; CAPELLA, S.O.; COSTA, A.L.; BRUHN, R.P.; NOBRE, M.O. Evaluation of cognitive dysfunction syndrome in dogs using an observational questionnaire. Semina: Ciênc. Agrár., v.40, n.5, p.2235-2246, 2019.

MAJZOUB, J.A. Corticotropin-releasing hormone physiology. Eur. Jour. End. p. S71-S76, 2006.

PAZ, J.E.G.; MACHADO, G.; COSTA, F.V.A. Fatores relacionados a problemas de comportamentos de gatos. Pesq. Vet. Bras. v.11, n.37, p.1336-1340, 2017.

QUESSADA, A.M.; BARBOSA, E.L.; NUNES, J.A.R.; OLIVEIRA, F.S.; ÚLTIMO, A.B.SUGAUARA, E.Y. Perfil de proprietários de cães nos municípios de Teresina (Brasil). Arq. ciê. Vet. e 45 Zoo. UNIPAR, v. 17, n. 3, p.173-175, 2014.

SCHOT, A.A.W-V.; DAY C.; MORTON, J.M.; RAND, J.; PHILLIPIS, C.J.C. Risk factors for behavior problems in cats presented to na Australian companion animal behavior clinic. Jour. of. Vet. Behav. v.14, p.34-40, 2016.

SOUZA, C.C.F.; MEDEIROS, M.A. Fatores de risco e transtornos comportamentais concomitantes em cães de companhia com medo exagerado a sons. Rev.Bras. Med. Vet., v.38, n.2, p.175-182, 2016.

WESTROPP J.L.; Kass PH and Buffington CA. Evaluation of the effects of stress in cats with idiopathic cystitis. Am J Vet Res, n. 67, p.731-736. 2006. 